\title{
STUDY OF CORN OPTIMIZATION IRRIGATION MODEL BY GENETIC ALGORITHMS
}

\author{
Bing Zhang ${ }^{1, *}$, ShouQi Yuan ${ }^{2}$, JianSheng Zhang ${ }^{1}$, Hong Li $^{2}$ \\ ${ }^{1}$ Changzhou institute of technology, Chang Zhou, China \\ ${ }^{2}$ Research Center of Fluid Machinery Engineering and Technology, JiangSu University, \\ Zhenjiang, China \\ *Corresponding author, Email: jiangsudaxuezb@163.com, Mobilephone: +86-13616110031
}

\begin{abstract}
Many factors affecting irrigation model, including irrigation water volume, crop water requirement, production function of irrigation water, rainfall, soil water balance, water sensitive index in different stages of crop growth, the grain market price, irrigation water price, minimum yield, irrigation cost etc are considered. Then a multi-constraints and non-linear optimization irrigation model based on the maximal profit of irrigation water volume is set up, which is adaptive to our national conditions, and the real number encoding space of the model is searched by the powerful searching ability of genetic algorithm. The results show that this model can solve the optimization irrigation problem of summer corn, and genetic algorithm has very perfect searching function, and the optimal solution of the model can be found in very short time.
\end{abstract}

Keywords: genetic algorithms, real number encoding, Jensen model, objective function, optimization irrigation

\section{INTRODUCTION}

Nowadays, water resource becomes more and more scarce in many regions due to increasing demand, and the water needed by agricultural irrigation can't be increased any more, so how to allocate the limited water supply effectively over different stages of crop growth, and how to control 
the quantity precisely for the maximum return from crop become the focus of agricultural irrigation engineering. With the sustainable agriculture developing, the key research on agricultural irrigation diverts from maximum yield to maximum return from crop with the same irrigation water volume.

It has always been a difficult problem to find optimal or near optimal solutions of irrigation model with traditionally dynamic optimization technology because of multi-factors, uncertainty and non-linearity in the model. To gain the optimal solutions, scientists adopt the dynamic programming stepwise approach (DPSA) and nonlinear programming (NLP), namely, a multi-stage decision-making process How to allocate the limited water supply over different stages of growth. But DPSA carries no guarantee that an optimum solution will be converged under any circumstance; and NLP is seldom used because of its nonlinearity.

Genetic Algorithms (GAs), as a kind of new global optimization search method, have many remarkable characteristics. They are computationally simple, adaptive and robust optimization techniques. GAs are based on the principles of population genetics and, constitute a special class among adaptive algorithms. They combine the adaptive process in nature with functional optimizations by simulating the selection of the best performing individuals in the populations. GAs are the iterative search method of "survival and detection" and start with an initial random population, then allocate initial solution to regions of the encoding search space. Among them, three main operators of GAs are selection, crossover, mutation; key content of GAs includes parameter encoding, the initial population settings, design of fitness function, penalty function, design of genetic operating and control parameters settings.

Previous studies on irrigation model have used binary encoding genetic algorithms. Necati Canpolat (1997) solves the seasonal irrigation scheduling using GAs. The goal of his irrigation model is finding the maximum net return. An irrigation schedule consists of a sequence dates on which water is to be applied to the crop, and for each date an amount of water to apply. The crop growth season of winter wheat is about 250 days, so the scheduling presents $2^{250}$ different possible irrigation decisions. Thus, the solution space for this problem is $2^{250}$. At the same time, different genetic parameters are established for result comparison, and then get a group of best genetic parameters. But this model has not considered the other essential constraints, just considers crop yield and water supply. Besides, because the water irrigated each time is certain, it is impractical to be used to irrigation practice. Fu Qiang et al. (2003) set up the irrigation system of rice irrigated by well under deficit irrigation in SanJiang Plain using the real code acceleration genetic algorithm (RAGA) with the multidimensional dynamic planning (DP), and have made satisfactory results. Jose Fernando Ortega Alvarez et al. (2004) set up irrigation scheduling model which gaining the 
optimum irrigation water volume of different crops using binary encoding genetic algorithms. K. Srinivasa Raju and D. Nagesh Kumar (2004) study an irrigation model on the basis of maximum net return of many crops using GAs, and find that genetic algorithm is an effective tool to solve the optimization irrigation model, and get a group of optimum genetic algorithm parameters.

This article presents an irrigation model considered multi-factors and multi-constraints, and the model can be well solved by Genetic Algorithms.

\section{SEARCH PROCEDURE OF GAS}

In general, the objective function can be described as:

$$
\max F_{i}=f(x)+\in \sum_{j=1}^{k} \delta_{j}\left(\phi_{j}\right)^{2}
$$

where $F_{i}$ is fitness value, $f(x)$ is objective function value, $\mathrm{k}$ is total number of constraints, $\in$ is -1 for maximization and +1 for minimization, $\delta_{j}$ is penalty coefficient and $\phi_{j}$ is amount of violation. Once the problem is converted into an unconstrained problem, rest of the procedure remains the same. A detailed description of genetic algorithms is given by Deb (1999).

Search procedure of GAs is as follows:

Step 1, initialization: Setting initial evolution step $t=0$; setting the maximal evolution step $T$; randomly creating an initial population $P(0)$ including $M$ individuals.

Step 2, Individual evaluation: Using an evaluation function (eval (V) is employed in this study) to assess the fitness of each individual.

Step 3, Selection: Using a probabilistic selection process, the population for the next generation is formed. The parent individuals are selected based on their fitness. Individuals with higher fitness have a greater chance of contributing offspring. The selection mechanism plays an important role for searching towards better solutions.

Step 4, Crossover: Crossover involves the exchange of genetic material from two patents by randomly swapping parts of their chromosomes. Crossover provides a powerful exploration capability new individual for further evaluation within the hyper planes already represented in the population.

Step 5, Mutation: A mutation operator $p_{m}$ is applied to the population. By modifying one or more of the gene values of an existing individual, mutation crates new individuals, generally resulting in increased variability of the population. It insures that the probability of reaching any point in the search 
space is never zero. A new population $\mathrm{P}(\mathrm{t}+1)$ is produced from previous population $\mathrm{P}(\mathrm{t})$ by the operation of selection, crossover and mutation.

Step 6, Judgments of ending: if $\mathrm{t} \leq \mathrm{T}$, go to step 2; If $\mathrm{t}>\mathrm{T}$, individual of the highest fitness is determined as the optimal solution, and operations of GAs stop.

\section{IRRIGATION MODEL}

The net return per ha from crop is determined by input-output relationship. The crop net return to irrigation water is calculated as the crop yield multiplied by the selling price of the crop (some scientists have also considered byproduct's income, government's subsidy etc.). Input includes irrigating cost, fertilizer, agriculture chemicals, workforce's expenses and agricultural tax, etc. In this study, irrigation costs are the only variation costs of production to be considered, other inputs are assumed to be constant. The goal is to find a long-term irrigation schedule that will provide the maximum return for crop growth. The objective function, $f\left(Y_{a}, W\right)$, is the net return from crop:

$$
f\left(Y_{a}, W\right)=Y_{a} * P_{Y}-W * P_{w}-\sum_{j=i}^{m} C_{j}
$$

where $f=$ net margin (yuan $\left.h a^{-1}\right) ; Y_{a}=$ real harvested yield $\left(\mathrm{kg} h a^{-1}\right)$; $P_{Y}=$ selling price of the product (yuan $\mathrm{kg}^{-1}$ ); $W=$ total irrigation volume $\left(\mathrm{m} 3 h a^{-1}\right) ; P_{W}=$ price of irrigation water $\left(\right.$ yuan $\left.m^{-3}\right) ; \sum_{j=1}^{m} C_{j}=\operatorname{sum}$ of other crop investments (yuan $h a^{-1}$ ); j represents number of other crop investments.

The actual crop yield $Y_{a}$ and total irrigation volume $W$ in the nonlinear irrigation model mentioned above have strong coupling relation, and traditional irrigation is based on theory of soil water balance. Under deficit condition, some factors, such as deep leakage, surface runoff, can be neglected. Therefore, soil water balance can be simplified as:

soil water balance: $W_{i}=E T_{i}-P_{e i}$

where $W_{i}=$ irrigation volume in the growth stage $i(\mathrm{~mm}) ; P_{e i}=$ rainfall in the growth stage $i(\mathrm{~mm}) ; E T_{i}=$ actual evapotranspiration in the growth stage $i(\mathrm{~mm})$; 
Different irrigation volume in each stage of crop growth has a complicated effect on crop yield, In this study, a numerical model described below, crop water production function (1968) is employed, which is popularly used, to predict crop yield corresponding to given irrigation schedule.

$$
\text { Jensen model: } \frac{Y_{a}}{Y_{m}}=\prod_{i=1}^{n}\left(\frac{E T_{i}}{E T_{m i}}\right)^{\lambda_{i}}
$$

where $E T_{i}=$ actual evatransporation in stage $i(\mathrm{~mm}) ; E T_{m i}=$ maximal evatransporation in stage $i(\mathrm{~mm}) ; n=$ the stage number of crop growth divided; $\lambda_{i}=$ water sensitive index in stage $i ; Y_{m}=$ maximum crop yield under abundant irrigation $\left(\mathrm{kg} h a^{-1}\right)$

A multi-factors crop irrigation model can be gained by considering Eqns (2), Eqns (3), and Eqns (4), which involves irrigation water volume, rainfall, water sensitive index, grain price and price of agricultural irrigation water influenced by market. What's more, the model Subjects to many constraints.

Irrigation model:

$$
f\left(Y_{a}, W\right)=Y_{m} \prod_{i=1}^{n}\left(\frac{W_{i}+P_{e i}}{E T_{m i}}\right)^{\lambda_{i}} P_{Y}-P_{W} \sum_{i=1}^{n} W_{i}-\sum_{j=1}^{m} C_{j}
$$

Subject to:

Constraint of total irrigation volume: $W_{\min } \leq \sum_{i=1}^{n} W_{i} \leq W_{\max }$

Constraint of irrigation volume in each stage: $W_{i \min } \leq W_{i} \leq W_{i \max }$

Constraint of minimal crop yield (basic grain consumption): $Y_{a} \geq Y_{\min }$

\section{RESULTS AND DISCUSSION}

Using the experimental data from the experiment region plot in Libao, Shanxi, to solve and verify the irrigation-margin model mentioned above, to find out the optimum water distribution and maximal return from irrigation of summer corn under deficit and multi-constraints condition, 


\subsection{Model Parameter Settings}

Objective function:

$$
f\left(Y_{a}, W\right)=Y_{m} \prod_{i=1}^{n}\left(\frac{W_{i}+P_{e i}}{E T_{m i}}\right)^{\lambda_{i}} P_{Y}-P_{W} \sum_{i=1}^{n} W_{i}-\sum_{j=1}^{m} C_{j}
$$

$\max$

where $Y_{m}=$ maximum crop yield under abundant irrigation condition $\left(6500 \mathrm{~kg} \mathrm{ha} \mathrm{a}^{-1}\right) ; P_{Y}=$ selling price of corn $\left(0.9\right.$ yuan $\left.\mathrm{kg}^{-1}\right) ; P_{W}=$ price of irrigation water (6yuan $\mathrm{mm}^{-1}$ ); $\sum_{j=1}^{m} C_{j}=$ sum of other crop investments (1500yuan $h a^{-1}$ ).

Table 1. Water sensitive index, maximal water requirement and rainfall in each stage of summer corn in Huoquan, Shanxi in 1998

\begin{tabular}{lcccc}
\hline Growth stage & $\begin{array}{c}\text { Sowing- } \\
\text { jointing }\end{array}$ & $\begin{array}{c}\text { Jointing- } \\
\text { tasseling }\end{array}$ & $\begin{array}{c}\text { Tasseling- } \\
\text { grouting }\end{array}$ & $\begin{array}{c}\text { Grouting- } \\
\text { ripening }\end{array}$ \\
\hline Water sensitive index & 0.0992 & 0.2368 & 0.3926 & 0.2014 \\
Maximal evatransporation in & & & & \\
each stage $(\mathrm{mm})$ & 94.56 & 112.25 & 124.04 & 90.57 \\
Rainfall $(\mathrm{mm})$ & 70.2 & 52.1 & 47.5 & 35 \\
\hline
\end{tabular}

Constraint matrix of irrigation volume in each stage:

Constraints $=\left[\begin{array}{cc}10 & 30 \\ 15 & 80 \\ 25 & 100 \\ 20 & 80\end{array}\right] \quad(\mathrm{mm})$

Constraint of minimal crop yield: $Y_{a} \geq 3500 \mathrm{~kg}$

\subsection{GAs Parameters}

The search course of GAs is close related to settings of GAs parameters. Before searching, solution space should be encoded. In this study, real number encoding is chosen for the purpose of precision. Then find the fitness value for each individual. The parent individuals are selected based on their finesses. Individuals with higher fitness have a greater probability of contributing offspring. In the selection of solution, proportional selection 
(also called Roulette Wheel) is used; the way of crossover adopts one-point selection, involves the exchange of genetic material from two parents by randomly swapping parts of their chromosomes and formation of individuals. In order to improve the partial search capacity of genetic algorithms, maintain the diversity of the population and prevent the premature phenomenon, operator of mutation is utilized in the course of search. Because of the randomness of genetic operation such as selection, crossover and mutation, the individual with best fitness in the present population might be deleted. GAs adopts the tactic of saving best solution in the search course. Meanwhile, there are a lot of constraints in the model; GAs punish the solution dissatisfying constraints with penalty function.

GAs parameter settings: The way of encoding adopts real number encoding; number of individuals in the population is $M=20$; the biggest step is $\mathrm{T}=100$; the way of selection is proportional selection; the way of crossover is one-point crossover; crossover probability is $p_{c}=0.9$; mutation probability is $p_{m}=0.01$, penalty function, which is used to punish the solutions dissatisfying constraints, is $F^{\prime}(x)=0.3 F(x)$.

\subsection{GAs Solution and Analysis}

The experimental data of summer corn from experiment region in Libao, Shanxi under deficit irrigation is used to find out the optimal solution by GAs. The solution should promise the maximal return from irrigation and satisfy the constraints.

Figure 1, figure 2, and figure 3 show that at the beginning of the run, the optimal solution of the population is fairly low. As the number of generations pass, the optimal solution has a definite upward trend which

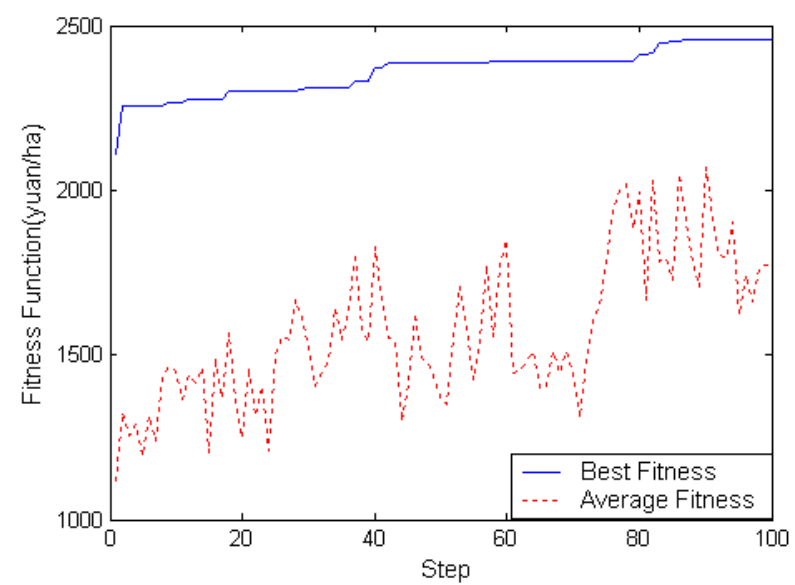

Figure 1. Best fitness and average fitness of model versus irrigation volume of $130 \mathrm{~mm}$ 


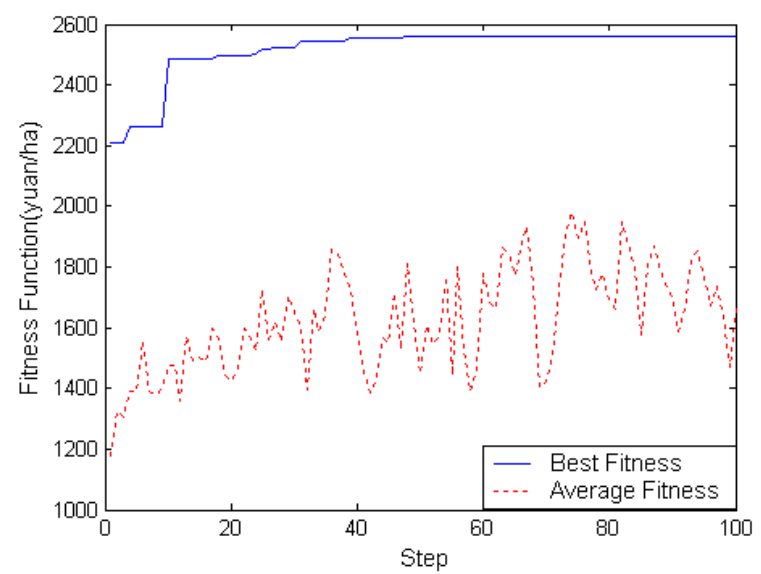

Figure 2. Best fitness and average fitness of model versus irrigation volume of $140 \mathrm{~mm}$

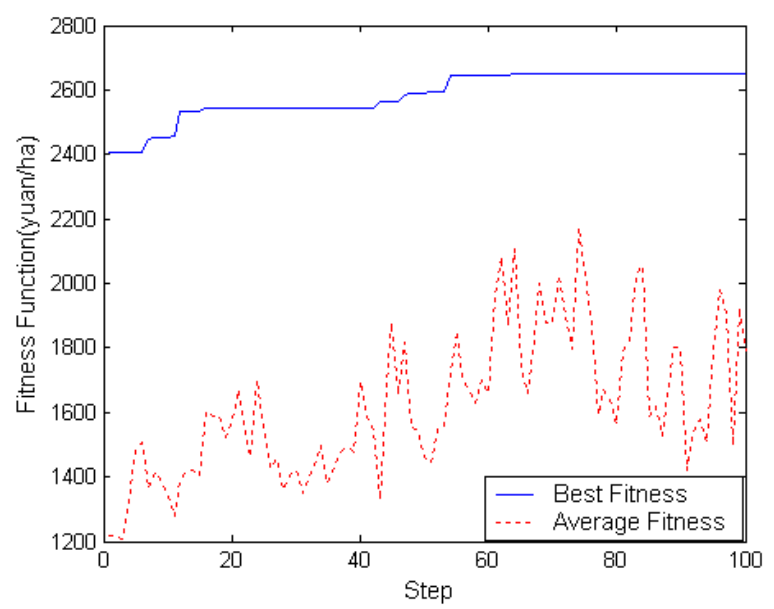

Figure 3. Best fitness and average fitness of model versus irrigation volume of $150 \mathrm{~mm}$

approaches the best of generation fitness levels. The increasing speed of optimal solution lowers. Again, When GAs reach certain step, fitness values keep invariable basically. According to the theory of GAs, at the initial stage, the average fitness of the population is fairly low. As the number of generations pass and as a result of GAs (selection, crossover, mutation), the average fitness fluctuates.

The average fitness of population is influenced by all fitness values of individuals; therefore, it can not be guaranteed that all individuals can be obviously improved in the genetic algorithms. Especially when the solution is out of the range of constraints, GAs will carry on corresponding 
punishment to fitness value according to penalty function; in other words, the fitness value change into a smaller one. So the average fitness of population fluctuates randomly.

In order to understand better the change of optimal solution and the influence of irrigation to return under different irrigation condition, the optimum solutions are compared in figure 4.

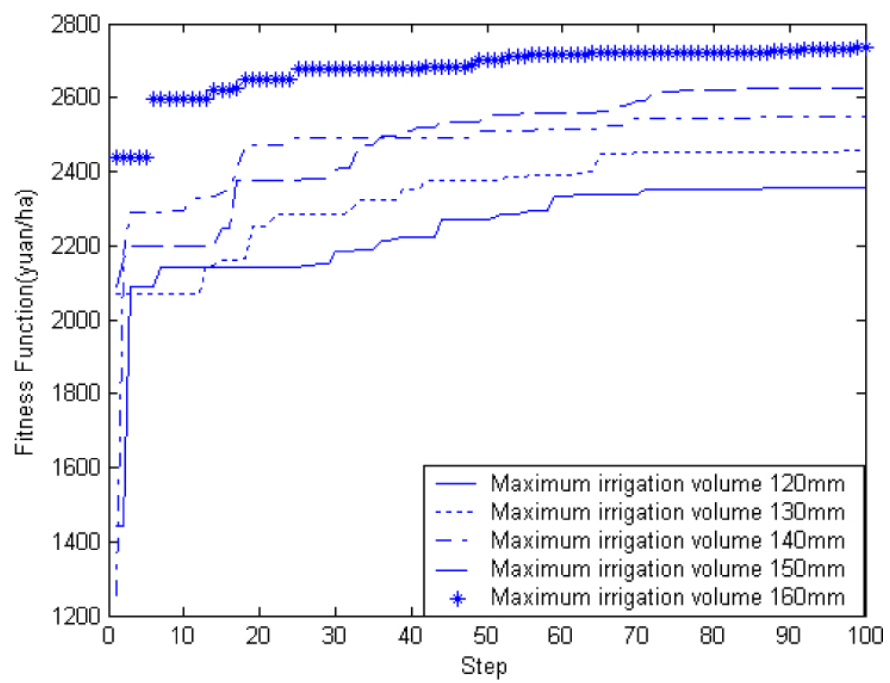

Figure 4. Comparison of optimal solution

From the comparison of optimal solution of irrigation volume under different irrigation level in figure 4, return from irrigation increases along with the increase of irrigation volume when under deficit irrigation condition. It obtains the maximum value when irrigation volume is 160 millimeters. When irrigation volume is 120 millimeters, it is the shallest.

From table 2, when the net return from corn is maximal, the optimum distribution of irrigation volume and corn yield response to irrigation schedule. Within certain irrigation range, under deficit irrigation condition, GAs choose upper limit of supplied irrigation water first. Since the water sensitive index in tasseling-grouting stage is the biggest, the proportion of irrigation volume is correspondingly the biggest, while the proportion in sowing - jointing stage is the smallest. GAs consider the influence of water sensitive index within certain irrigation range, which can be proved in table 2 . With the increase of irrigate volume, net return and yield increase at the same time. 
Table 2. Optimal distribution of irrigation volume, corn yield and net return under different irrigating condition

\begin{tabular}{|c|c|c|c|c|c|c|c|}
\hline \multirow{2}{*}{$\begin{array}{l}\text { Irrigation } \\
\text { volume } \\
(\mathrm{mm})\end{array}$} & \multicolumn{4}{|c|}{$\begin{array}{l}\text { Optimal distribution of limited irrigation water } \\
\text { supply in different stage of growth }(\mathrm{mm})\end{array}$} & \multirow{2}{*}{$\begin{array}{l}\text { Total } \\
\text { irrigation } \\
\text { volume } \\
(\mathrm{mm})\end{array}$} & \multirow{2}{*}{$\begin{array}{l}\text { Crop } \\
\text { yield } \\
\mathrm{kg} / \mathrm{ha}\end{array}$} & \multirow{2}{*}{$\begin{array}{c}\text { Net } \\
\text { return } \\
\text { Yuan/ha }\end{array}$} \\
\hline & $\begin{array}{l}\text { Sowing- } \\
\text { jointing }\end{array}$ & $\begin{array}{l}\text { Jointing- } \\
\text { tasseling }\end{array}$ & $\begin{array}{l}\text { Tasseling- } \\
\text { grouting }\end{array}$ & $\begin{array}{l}\text { Grouting- } \\
\text { ripening }\end{array}$ & & & \\
\hline $100-110$ & 10 & 28.6 & 36.5 & 34.6 & 109.7 & 4813 & 2173.5 \\
\hline $110-120$ & 10 & 33.7 & 56.2 & 20.1 & 120 & 5060.4 & 2334.3 \\
\hline $120-130$ & 10.1 & 30.8 & 64.7 & 24.3 & 129.9 & 5256.2 & 2450.6 \\
\hline $130-140$ & 10 & 20.4 & 82.6 & 27 & 140 & 5442.8 & 2558.5 \\
\hline $140-150$ & 10 & 16.7 & 77.6 & 45.6 & 149.9 & 5582.2 & 2624.2 \\
\hline $150-160$ & 10 & 26.1 & 91.1 & 32.8 & 160 & 5783.9 & 2745.5 \\
\hline $160-170$ & 10.7 & 27.5 & 94.2 & 37.6 & 170 & 5945.7 & 2831.1 \\
\hline
\end{tabular}

Figure 5, figure 6 and figure 7 illustrate that with the increase of irrigation water volume under deficit irrigation, net return and yield increase at the same time, but the net return per mm decreases, which complies with law of diminishing marginal utility. It can be predicted that, as the search continued, the yield will not increase any more when the corns are abundantly irrigated.

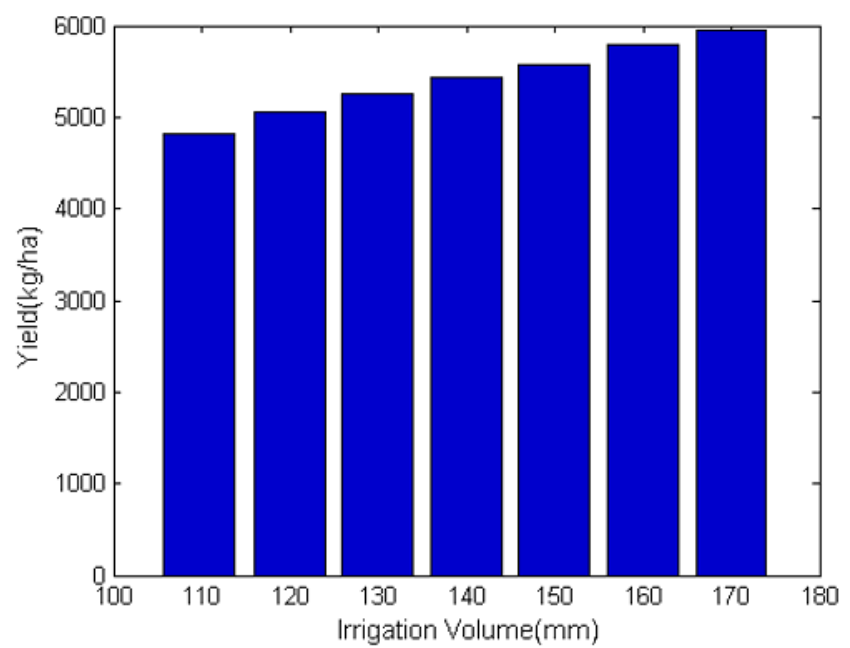

Figure 5. Relation between irrigation and yield 


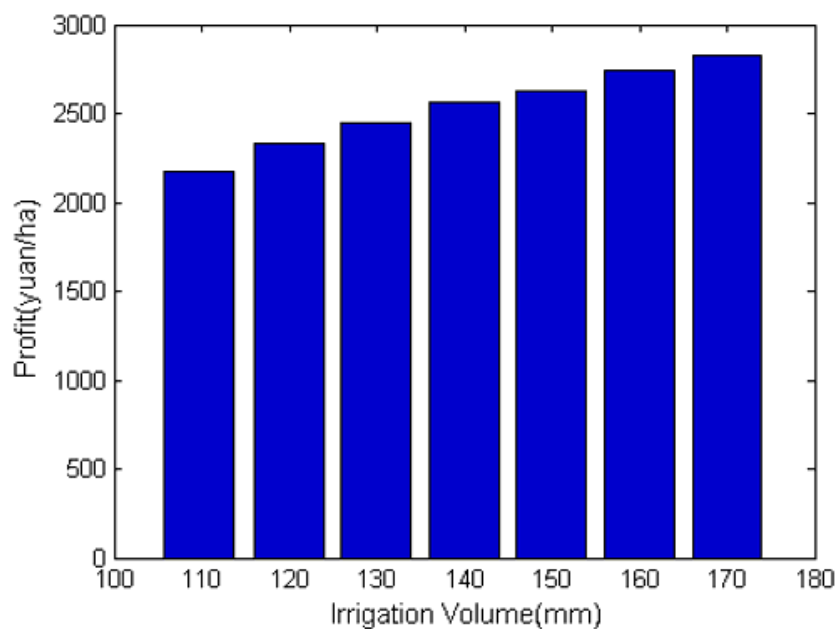

Figure 6. Relation between irrigation volume and profit

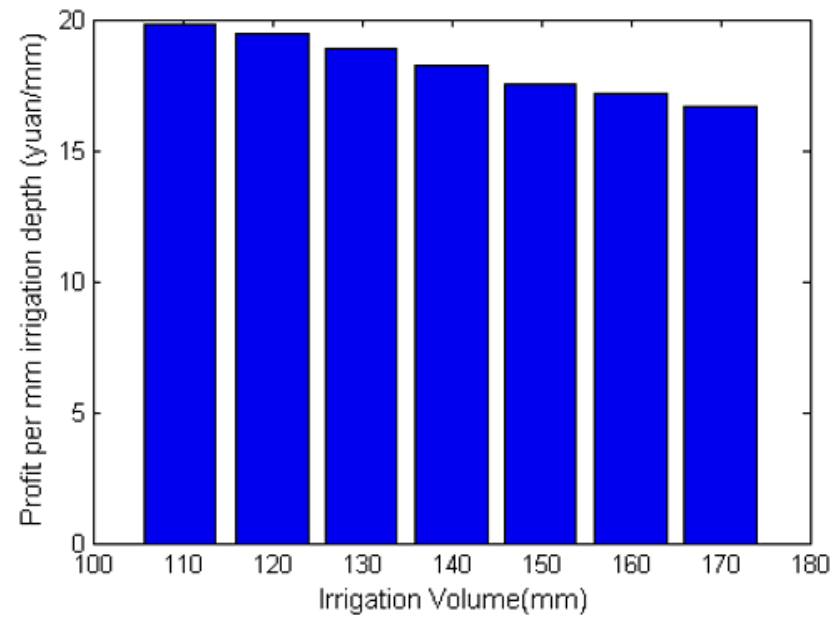

Figure 7. Relation between irrigation volume and profit per $\mathrm{mm}$ irrigation volume

\section{CONCLUSIONS}

(1) A multi-constraints and non-linear irrigation-return model is presented in this article, involving many factors, such as rainfall, water sensitive index, selling price of grain and irrigation water price, and combines soil water balance, production function of irrigation water. This irrigation model can meet the need of present agricultural irrigation practice of our country. 
(2) The irrigation model solved by the advanced global optimization search algorithm-genetic algorithm offers several advantages over the classical techniques such as DPSA and NLP. The classical techniques can't find the optimal or near optimal solution. By comparison, genetic algorithms perform well, especially in solving the uncertain and nonlinear model.

(3) The results indicate that we can easily allocate the limited water supply using this model, and it totally accords with the theory of genetic algorithms. Under deficit irrigation, when the optimum distribution of water satisfies the multi-constraints term, crop should be irrigated as much as possible, and irrigation water should be used more in the stage in which its water sensitive index is big. The crop yield and net return increase with the increase of the water irrigated, but the net return per unit decrease. These qualitative and quantitative results indicate that the model and genetic algorithms used here can apply in the nonlinear model and have good prospect.

\section{REFERENCES}

Abedalrazq F. Khalil, D. Nagesh Kumar. 2002 'Use of Artificial Neural Networks in Canal Irrigation Management', Utah State University, America.

Jose Fernando Ortega Alvarez. 2004, 'An Economic Optimization Model for Irrigation Water Management', Irrigation science. 23, 61-75.

K. Srinivasa Raju, L. Duckstein. 2003, 'Multiobjective fuzzy linear programming for sustainable irrigation planning: an Indian case study', Soft Computing. 7, 412-418.

K. Srinivasa Raju. 2004, 'Irrigation Planning Using Genetic Algorithms', Water Resources Management. 18, 163-176.

Kuo, S.F., Merkley, G.P. and Liu, C.W. 2000, 'Decision support for irrigation project planning using a genetic algorithm', Agri. Water Manage. 45, 243-266.

N.S. Raghuwanshi, W.W. Wallender. 1999, 'Forecasting and optimizing furrow irrigation management decision variables'. Irrigation Science. 19, 1-6.

Necati Canpolat. 1997, 'Optimization of Seasonal Irrigation Scheduling by Genetic Algorithms' A dissertation presented for the PHD. Oregon State University. America.

Paul Dominic Colaizzi. 2001, 'Ground Based Remote Sensing for Irrigation Management in Precision Agriculture ', A dissertation presented for the PHD. The university of Arizona. America.

Radwan A. Al-Weshah. 2000, 'Optimal use of irrigation water in the Jordan valley: A case study', Water Resources Management. 14, 327-338.

Renato Silvio da Frota Ribeiro. 1998, 'Fuzzy logic based automated irrigation control system optimized via neural networks', A dissertation presented for the PHD. The university of Tennessee. America.

Zhang Bing, Yuan Shouqi, Cheng Li. 2004 'Study on the Optimized Distribution Model of the Limited Irrigation Volume on the Basis of MATLAB'. 2004 CIGR International Conference. Beijing. 